International Journal of Pure and Applied Mathematics

Volume $91 \quad$ No. 2 2014, 143-153

ISSN: 1311-8080 (printed version); ISSN: 1314-3395 (on-line version)

url: http://www.ijpam.eu

doi: http://dx.doi.org/10.12732/ijpam.v91i2.1

\title{
WEAKLY BIASED MAPS AS A GENERALIZATION OF OCCASIONALLY WEAKLY COMPATIBLE MAPS
}

\author{
P.P. Murthy ${ }^{1 \S}$, M.R. Singh ${ }^{2}$, L.S. Singh ${ }^{3}$ \\ ${ }^{1}$ Department of Pure and Applied Mathematics \\ Guru Ghasidas Vishwavidyalaya (A Central University) \\ Koni, Bilaspur (Chhattisgarh), 495 009, INDIA \\ ${ }^{2}$ Department of Mathematics \\ Manipur University \\ Canchipur, 795003, Imphal, INDIA \\ ${ }^{3}$ Department of Mathematics \\ D.M. College of Teacher Education \\ Imphal, 795001, INDIA
}

\begin{abstract}
In this paper, we characterize the notion of weakly biased maps as a generalization of occasionally weakly compatible(owc) maps and establish common fixed point theorems for two pairs of weakly biased (resp. of type (A)) by using property (E.A.), without appealing continuity and completeness of space. The results improve and extend Theorem 2.11 in [12] and Theorem 3.5 in $[10]$.
\end{abstract}

AMS Subject Classification: 47H10, 54H25

Key Words: metric space, fixed point, property (E.A.), weakly compatible maps, owc maps, weakly biased maps, weakly biased maps of type (A)

\section{Introduction}

The concept of weak commutativity, compatibility, non-compatibility and weak compatibility were used as a sharper tool for the existence of common fixed

Received: August 13, 2012

(C) 2014 Academic Publications, Ltd.

$\S$ Correspondence author url: www.acadpubl.eu 
points for pair of contractive maps satisfying certain conditions on metric space (see e.g. [3], [4]). Generalizing the concept of commuting maps [3], Sessa [11] introduced the notion of weakly commuting maps. Jungck generalized this idea by weakening the concept introduced by Sessa[11] and introduced the concept of compatible maps [4] and then to weakly compatible maps [5]. Jungck, Murthy and Cho [6] introduced the concept of compatible maps of type(A) independent from compatible introduced by Jungck[4]. Al-Thagafi and Shahzad [2] introduced the concept of occasionally weakly compatible, a very general notion of weakly compatible. On the other hand, in 1995, Jungck and Pathak [7] coined a less restrictive notion of biased maps and weakly biased maps as a generalization of compatible maps. It is noted that compatible maps are biased maps, but the converse is not true [7]. In 1998, Pathak, Cho and Kang [10] weakened the condition of compatible maps of type (A) to that of biased maps of type (A) and weakly biased maps of type (A); and showed the existence of solutions of nonlinear integral equations. It is seen that compatible maps of type (A) are biased maps of type (A), but the converse is not necessarily true (see [10]). Recently, Singh and Mahendra [12] characterized biased concepts (viz, weakly biased, weakly biased of type (A)) with that of weakly compatible.

The main objective of this paper is to characterize the concept of weakly biased maps as a generalization of occasionally weakly compatible maps and to obtain common fixed point theorems for two pairs of weakly biased maps by using the property (E.A.); wherein the assumptions viz, continuity and completeness of space are waived. Our results complement, extend and unify many results of [6], [9], [10], [12], etc.

We begin with recalling some definitions needed in the paper:

Let $A$ and $S$ be two self-maps of a metric space $(\mathrm{X}, \mathrm{d})$ and $\mathrm{C}(\mathrm{A}, \mathrm{S})$ the set of coincidence points of $A$ and $S$, i.e. $C(A, S)=\{x: A x=S x\}$.

Definition 1. [1] $A$ and $S$ satisfy property (E.A.) if there exists a sequence $\left\{x_{n}\right\}$ in $X$ such that $\lim _{n \rightarrow \infty} A x_{n}=\lim _{n \rightarrow \infty} S x_{n}=t$ for some $t \in X$.

Definition 2. [5] $A$ and $S$ are said to be weakly compatible if they commute at coincidence points, i.e. $S A t=A S t$ for all $t \in C(A, S)$.

Definition 3. [2] $A$ and $S$ are said to be occasionally weakly compatible (shortly owc) iff there is a point $t$ in $X$ such that $A t=S t$ at which $A$ and $S$ commute, i.e. $S A t=A S t$ for all $t \in C(A, S)$.

Clearly, weakly compatible maps are occasionally weakly compatible, but the converse is not true.

Definition 4. [7] $A$ and $S$ are said to be weakly $S$-biased iff $A t=S t$ 
implies $d(S A t, S t) \leq d(A S t, A t)$.

The pair $\{A, S\}$ is weakly A-biased if the roles of $A$ and $S$ are interchanged in this definition. It is noted that biased maps are weakly biased (see Prop. 1.1 of [7]). From [12], it is seen that weakly compatible maps are weakly biased, but implication is not reversible.

Definition 5. $A$ and $S$ are said to be weakly $S$-biased of type (A) if $A t=S t$ implies $d(S S t, A t) \leq d(A S t, S t)$.

To be weakly A-biased of type (A), A and S have to satisfy, in lieu of inequality $d(A A t, S t) \leq d(S A t, A t)$. in this definition. In [12], it is clear that the notions of weakly biased and weakly biased of type (A) are invariant. It is noted that biased of type (A) is weakly biased of type (A) [10]. Also they are distinct in nature (see Ex. 2.11, 2.12 of [10]).

Throughout this paper, $\mathbb{N}$ denotes the set of positive integers, $R^{+}$the set of non-negative real numbers, $\mathrm{R}$ the set of real numbers and $S(p, r)$ the open sphere with center $p$ and radius $r$, i.e. $S(p, r)=\{x: d(x, p)<r\}$.

\section{Main Theorems}

We deal with characterization for weakly biased in Section 1 and existence of common fixed point theorems in Section 2. In our results, the maps satisfying the property (E.A.) are not taken to be continuous at the fixed point. We employ weakly biased (resp. of type (A)) in lieu of commuting maps, various types of compatible maps. The completeness of the space is replaced by the closeness of one of the ranges of the maps.

Before going into the Proofs of the propositions, we illustrate the following example as a manifestation of the fact that weakly $A$-biased does not imply weakly $S$-biased of type (A).

Example 6. Let $X=[0,1]$ with the usual metric $d$ i.e. $d(x, y)=|x-y|$. Define the maps $A, S: X \rightarrow X$ by

$A(x)=\left\{\begin{array}{ll}1-2 x & ; \text { if } x \in\left[0, \frac{1}{2}\right) \\ \frac{1}{3} & ; \text { if } x \in\left[\frac{1}{2}, 1\right]\end{array}\right.$ and $S(x)= \begin{cases}2 x & ; \text { if } x \in\left[0, \frac{1}{2}\right) \\ 0 & ; \text { if } x \in\left[\frac{1}{2}, 1\right]\end{cases}$

respectively. We have $A x=S x$ iff $x=\frac{1}{4}$ So, we obtain $A\left(\frac{1}{4}\right)=\frac{1}{2}=S\left(\frac{1}{4}\right)$.

Since, $S S\left(\frac{1}{4}\right)=0, S A\left(\frac{1}{4}\right)=0$ and $A S\left(\frac{1}{4}\right)=\frac{1}{3}$, we have

$$
\begin{aligned}
& d\left(A S\left(\frac{1}{4}\right), A\left(\frac{1}{4}\right)\right)=\frac{1}{6} ; \\
& d\left(S A\left(\frac{1}{4}\right), S\left(\frac{1}{4}\right)\right)=\frac{1}{2} ;
\end{aligned}
$$




$$
d\left(S S\left(\frac{1}{4}\right), A\left(\frac{1}{4}\right)\right)=\frac{1}{6} .
$$

Therefore, we conclude that the pair $\{A, S\}$ is weakly A-biased, but not weakly S-biased of type (A).

We need the following lemma to our Proposition.

Lemma 7. Let $\{A, S\}$ be a pair of self-maps of a metric space $(X, d)$. If $x \in C(A, S)$ then the pair $\{A, S\}$ is either weakly A-biased or weakly $S$-biased.

Proof. Since $x \in C(A, S)$ we have $A x=S x$. Two cases arise : (i) $x \in$ $C(A, S)$ such that $A S x=S A x$ and (ii) $x \in C(A, S)$ such that $A S x \neq S A x$.

Case I: If $x \in C(A, S)$ such that $A S x=S A x$ then

$$
d(S A x, S x) \leq d(S A x, A S x)+d(A S x, A x)+d(A x, S x) .
$$

Therefore, $d(S A x, S x) \leq d(A S x, A x)$.

Similarly, by interchanging $A$ and $S$ in the above, we obtain $d(A S x, A x) \leq$ $d(S A x, S x)$.

Case II: If $x \in C(A, S)$ such that $A S x \neq S A x$ then either $A S x<S A x$ or $S A x<A S x$.

Suppose that $r_{1}$ and $r_{2}$ are two positive real numbers such that $A S x<r_{1}<$ $S A x<r_{2}$. Then we obtain two open spheres $S\left(A x, r_{1}\right)$ and $S\left(S x, r_{2}\right)$ such that $S\left(A x, r_{1}\right) \subseteq S\left(S x, r_{2}\right)$. Therefore, $d(A S x, A x) \leq d(S A x, S x)$.

On the other hand, suppose that $\delta_{1}$ and $\delta_{2}$ are two positive real numbers such that $S A x<\delta_{1}<A S x<\delta_{2}$. Then there exists two open spheres $S\left(S x, \delta_{1}\right)$ and $S\left(A x, \delta_{2}\right)$ such that $S\left(S x, \delta_{1}\right) \subseteq S\left(A x, \delta_{2}\right)$ So, $d(S A x, S x) \leq d(A S x, A x)$.

From both cases, we see that if $x \in C(A, S)$ then either $d(A S x, A x) \leq$ $d(S A x, S x)$ or $d(S A x, S x) \leq d(A S x, A x)$. Hence, if $x \in C(A, S)$ then the pair $\{A, S\}$, is either weakly A-biased or weakly S-biased.

The following proposition conveys that occasionally weakly compatible relates directly to one of the weakly A- biased and weakly S-biased.

Proposition 8. Let $\{A, S\}$, be a pair of self-maps of a metric space $(X, d$.) If the pair $\{A, S\}$, is occasionally weakly compatible, then it is either weakly A-biased or weakly S-biased.

Proof. Since the pair $\{A, S\}$, is occasionally weakly compatible, there exists a point $x \in X$ such that $A x=S x$ at which $A S x=S A x$. Moreover, there may exist a point $y \in X$ such that $A y=S y$ at which $A S y \neq S A y$. By Lemma[7, the pair $\{A, S\}$, is either weakly A-biased or weakly S-biased. Hence, occasionally 
weakly compatibility of $\{A, S\}$, implies either weakly A-biased or weakly Sbiased.

We furnish an example to establish the utility of our proposition $[8$.

Example 9. Let $X=[1, \infty)$ endowed with the usual metric $d(x, y)=\mid$ $x-y \mid$. Define maps $A, S: X \rightarrow X$ by $A x=3 x-2$. and $S x=x^{2}$.

Now, we have $A x=S x$ iff $x=1$ or $x=2$ and so, $A(1)=1=S(1)$ and $A(2)=4=S(2)$. Also, we obtain $A S(1)=1=S A(1)$ but $A S(2) \neq S A(2)$ as $A S(2)=10$ and $S A(2)=16$. Therefore, the pair $\{S, A\}$, is occasionally weakly compatible.

Since $d(A S(2), A(2))=6$ and $d(S A(2), S(2))=10$, we have $d(A S(2), A(2)) \leq$ $d(S A(2), S(2))$. One can substantiate that $d(A S(1), A(1)) \leq d(S A(1), S(1))$. Therefore, the pair $\{A, S\}$ is weakly A-biased. Hence, the pair $\{A, S\}$ is occasionally weakly compatible as well as weakly A-biased.

However, the converse of the above proposition is not necessarily true. The following example reveals the fact that the pair $\{A, S\}$ is weakly A-biased but not occasionally weakly compatible.

Example 10. Let $X=[1, \infty)$ endowed with the usual metric. Define maps $A, S: X \rightarrow X$ by $A x=5 x$ and $S x=x^{2}+6$. Now, we have $A x=S x$ iff $x=2$ or $x=3$ and so, $A(2)=10=S(2)$ and $A(3)=15=S(3)$.

Since $A S(2)=A(10)=50, S A(2)=S(10)=106, A S(3)=A(15)=75$ and $S A(3)=S(15)=231$.

We have $d(A S(2), A(2))=40 ; d(S A(2), S(2))=96 ; d(A S(3), A(3))=60$ and $d(A S(3), A(3))=216$.

So, we have $d(A S(2), A(2)) \leq d(S A(2), S(2))$ and $d(A S(3), A(3)) \leq S A(3)$, $S(3))$.

But, $A S(2) \neq S A(2)$ and $A S(3) \neq S A(3)$. Therefore, the pair $\{A, S\}$ is weakly A-biased but not occasionally weakly compatible. One can verify from example(2.2) that the pair A, S is weakly S-biased but not occasionally weakly compatible.

We conclude this sub-section by noting that the concept of weakly biased maps appears to be an effective generalization of occasionally weakly compatible maps of metric space. 


\section{Common Fixed Point Theorems}

Let $F$ be a family of all function $\phi:\left(R^{+}\right)^{5} \rightarrow R^{+}$such that $\phi$ is upper semicontinuous, non-decreasing in each coordinate variable and, for any $t>0$, $\phi(t, t, 0, \alpha t, 0) \leq \beta t, \phi(t, t, 0,0, \alpha t) \leq \beta t$, where $\beta=1$ for $\alpha=2$ and $\beta<1$ for $\alpha<2, \gamma(t)=\phi\left(t, t, a_{1} t, a_{2} t, a_{3} t\right)<t$, where $\gamma: R^{+} \rightarrow R^{+}$is a mapping and $a_{1}+a_{2}+a_{3}=4$.

We prove the following lemma before going to main theorem.

Lemma 11. Let $A, B, S$ and $T$ be self-maps of a metric space $(X, d)$ satisfying the following conditions:

(i) $A X \subset T X$ and $B X \subset S X$;

(ii)

$$
\begin{aligned}
& \quad d^{2 p}(A x, B y) \leq \phi\left(d^{2 p}(S x, T y), d^{p}(S x, A x) \cdot d^{p}(T y, B y),\right. \\
& d^{p}(S x, B y) \cdot d^{p}(T y, A x), d^{p}(S x, A x) \cdot d^{p}(T y, A x), d^{p}(S x, B y) \cdot d^{p}(T y, B y), \\
& \text { for all } x, y \in X \text { where } p \geq 1 \text { and } \phi \in F ;
\end{aligned}
$$

(iii) one range of the maps $A, B, S$ and $T$ is a closed subspace of $X$;

(iv) one of the pairs $\{A, S\}$ and $\{B, T\}$ satisfies the property (E.A.)

Then each of the pairs $\{A, S\}$ and $\{B, T\}$ has a coincidence point.

Proof. Suppose that the pair $\{B, T\}$ satisfies the property (E.A.). Then there exists a sequence $\left\{x_{n}\right\}$ in $X$ such that $\lim _{n \rightarrow \infty} B x_{n}=\lim _{n \rightarrow \infty} T x_{n}=t$ for some $t \in X$. Since $B X \subset S X$, there exists a sequence $\left\{y_{n}\right\} \subset X$ such that $B x_{n}=S y_{n}$ So, $\lim _{n \rightarrow \infty} S y_{n}=t$. We now show that $\lim _{n \rightarrow \infty} A y_{n}=t$.

Since $d\left(A y_{n}, t\right) \leq d\left(A y_{n}, B x_{n}\right)+d\left(B x_{n}, t\right)$, it is enough to show that

$$
\lim _{n \rightarrow \infty} d\left(A y_{n}, B x_{n}\right)=0 .
$$

If not, then there exists a real number $\epsilon>0$ such that $\lim _{n \rightarrow \infty} d\left(A y_{n}, B x_{n}\right)=\epsilon$. This assures that there exists a subsequence $\left\{y_{n_{k}}\right\}$ of $\left\{y_{n}\right\}$ in $X$ such that for each positive integer $k \geq n, \lim _{k \rightarrow \infty} d\left(A y_{n_{k}}, B x_{n_{k}}\right)=\epsilon$.

From $($ ii $)$, we have

$$
d\left(A y_{n_{k}}, B x_{n_{k}}\right) \leq\left[\phi \left(d^{2 p}\left(S y_{n_{k}}, T x_{n_{k}}\right), d^{p}\left(S y_{n_{k}}, A y_{n_{k}}\right) \cdot d^{p}\left(T x_{n_{k}}, B x_{n_{k}}\right),\right.\right.
$$




$$
\begin{aligned}
& d^{p}\left(S y_{n_{k}}, B x_{n_{k}}\right) \cdot d^{p}\left(T x_{n_{k}}, A y_{n_{k}}\right), \\
& d^{p}\left(S y_{n_{k}}, A y_{n_{k}}\right) \cdot d^{p}\left(T x_{n_{k}}, A y_{n_{k}}\right), \\
& \left.d^{p}\left(S y_{n_{k}}, B x_{n_{k}}\right) \cdot d^{p}\left(T x_{n_{k}}, B x_{n_{k}}\right)\right)^{1 / 2 p} .
\end{aligned}
$$

Taking $k \rightarrow \infty$ in the above inequality, we obtain $\epsilon \leq\left[\phi\left(0,0,0, \epsilon^{2 p}, 0\right)\right]^{1 / 2 p} \leq$ $\left[\gamma\left(\epsilon^{2 p}\right)\right]^{1 / 2 p}<\epsilon$ which is a contradiction.

Therefore, $\lim _{k \rightarrow \infty} d\left(A y_{n_{k}}, B x_{n_{k}}\right)=0$ and so, $\lim _{n \rightarrow \infty} d\left(A y_{n}, B x_{n}\right)=0$ which implies $\lim _{n \rightarrow \infty} A y_{n}=t$.

Suppose that $S X$ is a closed subspace of $X$. Then $t=S u$ for some $u \in X$. Subsequently, we have $\lim _{n \rightarrow \infty} A y_{n}=\lim _{n \rightarrow \infty} B x_{n}=\lim _{n \rightarrow \infty} S y_{n}=\lim _{n \rightarrow \infty} T x_{n}=t=S u$.

To prove that, $A u=t$ using (ii), we obtain

$$
\begin{aligned}
d\left(A u, B x_{n}\right) \leq & {\left[\phi \left(d^{2 p}\left(S u, T x_{n}\right), d^{p}(S u, A u) \cdot d^{p}\left(T x_{n}, B x_{n}\right),\right.\right.} \\
& d^{p}\left(S u, B x_{n}\right) \cdot d^{p}\left(T x_{n}, A u\right), d^{p}(S u, A u) \cdot d^{p}\left(T x_{n}, A u\right), \\
& \left.\left.d^{p}\left(S u, B x_{n}\right) \cdot d^{p}\left(T x_{n}, B x_{n}\right)\right)\right]^{1 / 2 p} .
\end{aligned}
$$

As $n \rightarrow \infty$ it follows that

$$
d(A u, t) \leq\left[\phi\left(0,0,0, d^{2 p}(A u, t), 0\right)\right]^{1 / 2 p} \leq\left[\gamma\left(d^{2 p}(A u, t)\right)\right]^{1 / 2 p}<d(A u, t) .
$$

This is contradiction and therefore, $A u=t$. Consequently, $u$ is a coincidence point of the pair $\{A, S\}$. From $A X \subset T X$ which gives $t \in T X$ we declare that there exists $v \in X$ such that $T v=t$.

To prove that $B v=t$, suppose that $B v \neq t$ By $(i i)$, we have

$$
\begin{aligned}
d(t, B v)= & d(A u, B v)=\left[\phi \left(d^{2 p}(S u, T v), d^{p}(S u, A u) \cdot d^{p}(T v, B v),\right.\right. \\
& d^{p}(S u, B v) \cdot d^{p}(T v, A u), d^{p}(S u, A u) \cdot d^{p}(T v, A u), \\
& \left.\left.d^{p}(S u, B v) \cdot d^{p}(T v, B v)\right)\right]^{1 / 2 p}=\left[\phi\left(0,0,0,0, d^{2 p}(t, B v)\right)\right]^{1 / 2 p} \\
\leq & {\left[\gamma\left(d^{2 p}(t, B v)\right)\right]^{1 / 2 p}<d(t, B v) }
\end{aligned}
$$

which means that $B v=t$. Therefore, the pair $\{B, T\}$ has a coincidence point $v$.

The same result holds if we suppose that one of $A X, B X$ and $T X$ is closed subspace of $X$.

Theorem 12. Let $A, B, S$ and $T$ be self-maps as in Lemma(11) satisfying $(i)-(i v)$. If the pairs $\{A, S\}$ and $\{B, T\}$ are weakly S-biased and weakly $T$ biased respectively, then $A, B, S$ and $T$ have a unique common fixed point. 
Proof. Assume that $S X$ is a closed subspace of $X$ and let $t, u$ and $v$ be as in Lemma(11). By that Lemma, $u$ and $v$ are coincidence points of the pairs $\{A, S\}$ and $\{B, T\}$ respectively.

Since the pair $\{A, S\}$ is weakly S-biased, $A u=S u$ implies $d(S A u, S u) \leq$ $d(A S u, A u)$ which gives $d(S t, t) \leq d(A t, t)$. On the other hand $A u=S u$ implies $A A u=A S u$ and $S A u=S S u$.

Subsequently, $d(S A u, A A u) \leq d(S A u, S u)+d(S u, A A u) \leq d(A S u, A u)+$ $d(A A u, A u)$ which implies $d(S t, A t) \leq 2 d(A t, t)$.

To show that $d(A t, t)=0$, suppose that $A t \neq t$.

From (ii), we have

$$
\begin{aligned}
d(A t, t)= & d(A t, B v) \leq\left[\phi \left(d^{2 p}(S t, T v), d^{p}(S t, A t) \cdot d^{p}(T v, B v), d^{p}(S t, B v) \cdot d^{p}(T v, A t),\right.\right. \\
& \left.\left.d^{p}(S t, A t) \cdot d^{p}(T v, A t), d^{p}(S t, B v) \cdot d^{p}(T v, B v)\right)\right]^{1 / 2 p} \\
= & {\left[\phi\left(d^{2 p}(S t, t), 0, d^{p}(S t, t) \cdot d^{p}(t, A t), d^{p}(S t, A t) \cdot d^{p}(A t, t), 0\right)\right]^{1 / 2 p} } \\
\leq & {\left[\phi\left(d^{2 p}(A t, t), 0, d^{2 p}(A t, t), 2 d^{2 p}(A t, t), 0\right)\right]^{1 / 2 p} } \\
= & {\left[\gamma\left(d^{2 p}(A t, t)\right]^{1 / 2 p}<d(A t, t) .\right.}
\end{aligned}
$$

which is a contradiction. Therefore, $A t=t$.

Further, since $d(S A u, S u) \leq d(A S u, A u)=d(A A u, A u)$, we have $d(S t, t) \leq$ $d(A t, t)$, which implies $d(S t, t)=0$. It follows that $S t=t$. Consequently, $t$ is a common fixed point of the pair $\{A, S\}$. Similarly, one can prove that $t$ is a common fixed point of the pair $\{B, T\}$. Thus, we conclude that $t$ is a common fixed point of $A, B, S$ and $T$.

If $z \in X$ is also a common fixed point of $A, B, S$ and $T$ with $t \neq z$, then using (ii), we have

$$
\begin{aligned}
d(t, z)= & d(A t, B z) \leq\left[\phi\left(d^{2 p}(S t, T z), d^{p} S t, A t\right) \cdot d^{p}(T z, B z), d^{p} S t, B z\right) \cdot d^{p}(T z, A t), \\
& \left.\left.\left.\left.d^{p} S t, A t\right) \cdot d^{p}(T z, A t), d^{p} S t, B z\right) \cdot d^{p}(T z, B z)\right)\right]^{1 / 2 p} \\
= & {\left[\phi\left(d^{2 p}(t, z), 0, d^{2 p}(t, z), 0,0\right)\right]^{1 / 2 p} } \\
\leq & {\left[\gamma\left(d^{2 p}(t, z)\right]^{1 / 2 p}<d(t, z) .\right.}
\end{aligned}
$$

This is a contradiction and therefore, $d(t, z)=0$, which implies $t=z$. This completes the proof.

From Theorem(12) we deduce the following corollary.

Corollary 13. ([12], Theorem 2.11) Let $A, B, S$ and $T$ be self-maps as in Lemma(11) satisfying (i), (iii), (iv) and the following

$$
d^{2 p}(A x, B y) \leq \alpha d^{2 p}(S x, T y)+\frac{\beta}{2^{p}} \max \left\{d^{p}(S x, A x) \cdot d^{p}(T y, B y),\right.
$$




$$
\begin{aligned}
& d^{p}(S x, B y) \cdot d^{p}(T y, A x), d^{p}(S x, A x) \cdot d^{p}(T y, A x), \\
& \left.d^{p}(S x, B y) \cdot d^{p}(T y, B y)\right\}
\end{aligned}
$$

for all $x, y \in X$ where $p \geq 1 ; \alpha, \beta>0$ and $\alpha+\beta<1$.

If the pairs $\{A, S\}$ and $\{B, T\}$ are weakly $S$-biased and weakly T-biased respectively, then $A, B, S$ and $T$ have a unique common fixed point.

It is to be noted that equation (3.1) is a special case of condition (ii) with $\phi\left(t_{1}, t_{2}, t_{3}, t_{4}, t_{5}\right)=\alpha t_{1}+\frac{\beta}{2^{p}} \max \left\{t_{2}, t_{3}, t_{4}, t_{5}\right)$ for all $t_{1}, t_{2}, t_{3}, t_{4}, t_{5} \in R^{+}$, where $p \geq 1 ; \alpha, \beta>0$ and then the above corollary follows immediately from Theorem(12). In lieu of a weakly biased in Theorem(12), we employ weakly biased of type (A) in the following theorem.

Theorem 14. Let $A, B, S$ and $T$ be self-maps as a Lemma(11) satisfying $(i)-(i v)$. If the pairs $\{A, S\}$ and $\{B, T\}$ are weakly S-biased of type (A) and weakly $T$-biased of type (A) respectively, then $A, B, S$ and $T$ have a unique common fixed point.

Proof. From [12], weakly biased and weakly biased of type (A) are invariant. Therefore, the result follows immediately from Theorem(14).

We obtain the following corollary.

Corollary 15. Let $A, B, S$ and $T$ be self-maps as in Lemma(11) satisfying (i), (iii), (iv) and (3.1). If the pairs $\{A, S\}$ and $\{B, T\}$ are weakly $S$-biased of type (A) and weakly T-biased of type (A) respectively, then $A, B, S$ and $T$ have a unique common fixed point.

Its proof follows from Theorem(14)

Example 16. Let $X=[0,1]$ endowed with the usual metric $d(x, y)=\mid$ $x-y \mid$ and define maps $A, B, S, T: X \rightarrow X$ by

$A(x)=B(x)= \begin{cases}\frac{x}{2} & ; \text { if } 0 \leq x<1 \\ 0 & ; \text { if } x=1\end{cases}$

$S(x)=T(x) \begin{cases}x & ; \text { if } 0 \leq x<1 \\ 1 & ; \text { if } x=1]\end{cases}$

respectively. Clearly $A X=B X=\left[0, \frac{1}{2}\right) \subset[0,1]=S X=T X$ is closed.

Consider $\phi\left(t_{1}, t_{2}, t_{3}, t_{4}, t_{5}\right)=\alpha t_{1}+\frac{\beta}{2^{p}} \max \left\{t_{2}, t_{3}, t_{4}, t_{5}\right)$ for all $t_{1}, t_{2}, t_{3}, t_{4}, t_{5}$ in $R^{+}$, where $p \geq 1 ; \alpha, \beta>0$ and $\alpha+\beta<1$. Then $\phi$ satisfies all the required conditions.

In particular, we take $\alpha=\frac{1}{3}$ and $\beta=\frac{1}{2}$.

Now, if $x, y \in[0,1)$ then $d^{2 p}(A x, B y)=\frac{1}{2^{2 P}} d^{2 P}(S x, T y)$, and if $x \in[0,1)$ and $y=1$ then $d^{2 p}(A x, B y)=d^{2 P}(S x, A x)$. 
Further if $x=1$ and $y=1$ then $d^{2 p}(A x, B y)=0$.

In all cases, we obtain

$$
\begin{aligned}
d^{2 p}(A x, B y) \leq & \frac{1}{3} d^{2 P}(S x, T y)+\frac{1}{4} \max \left\{d^{p}(S x, A x) \cdot d^{p}(T y, B y),\right. \\
& d^{p}(S x, B y) \cdot d^{p}(T y, A x), d^{p}(S x, A x) \cdot d^{p}(T y, A x), \\
& \left.d^{p}(S x, B y) \cdot d^{p}(T y, B y)\right\}
\end{aligned}
$$

Let $\left\{x_{n}\right\} \subset X$ be a sequence such that $A x_{n}, S x_{n} \rightarrow t$ for some $t \in X$. For this, let $x_{n} \rightarrow 0$ and $x_{n}>0$ for all $n \in N$. Then, $A x_{n}=\frac{1}{2} x_{n} \rightarrow 0$ and $S x_{n}=x_{n} \rightarrow 0=t$.

Now, we have $A x=S x$ iff $x=0$. So, we obtain $A(0)=0=S(0)$.

Since $S A(0)=S(0)=0$ and $A S(0)=A(0)=0$, we have $d(S A(0), S(0)) \leq$ $d(A S(0), A(0))$. Therefore, the pair $\{A, S\}$ is weakly S-biased. One can ascertain that the pair $\{B, T\}$ is weakly T-biased. Thus, all the conditions of Theorem(12) are satisfied and 0 is the unique common fixed point $A, B, S$ and $T$. One can verify that this example shows the validity of Theorem(14).

Remark 17. (1) Contractive condition (ii) is more general than that of Theorem 2.11[12].

(2) For $p=1$, contractive condition (ii) reduces to condition (3.2)(Theorem 3.5 of $[10])$.

(3) For $p=1$ and $A=B$ contractive condition (ii) reduces to condition (ii) (Theorem 2.3 of [9]).

\section{Acknowledgments}

First author(PPM) would like to thank to University Grants Commission, New Delhi, India for financial assistance through Major Research Project F.No. 42$32 / 2013$ (SR).

\section{References}

[1] M. Aamri, D.El Moutawakil, Some new common fixed point theorems under strict contractive conditions, J. Math. Appl. 270(2002), 181-188.

[2] M.A. Al-Thagafi, N. Shahzad, Generalized I-non expansive self maps and invariant approximations, Acta Math. Sinica 24(2008), no. 5, 867-876. 
[3] G. Jungck, Commuting mappings and fixed points, Amer. Math. Monthly 83(1976), 261-263.

[4] G. Jungck, Compatible mappings and common fixed points, Internat. J. Math. and Math. Sci. 9(1986), no. 4, 771-779.

[5] G. Jungck: Common fixed points for non continuous nonself maps on non metric spaces, Far East J. Math. Sci. 4(1996), no. 2, 199-215.

[6] G. Jungck, P.P. Murthy, Y.J. Cho: Compatible mappings of type (A) and common fixed points, Math. Japonica 38(1993), no. 2, 381-390.

[7] G. Jungck, H.K. Pathak, Fixed point via "biased maps", Proc. Amer. Math. Soc. 123 (1995), 2049-2060.

[8] S.M. Kang, B.E. Rhoades, Fixed points for four mappings, Math. Japonica 37 (1992), 1053-1059.

[9] H.K. Pathak, Weak* commuting mappings and fixed points, Indian J. Pure and Appl. Math. 17(1986), no. 2, 201-211.

[10] H.K. Pathak, Y.J. Cho, S.M. Kang, Common fixed points of biased maps of type (A) and applications, Internat. J. Math. and Math. Sci. Vol. 21, no. 4 (1998), 681-694.

[11] S. Sessa, On a weak commutativity condition in fixed point considerations, Publ. Inst. Math. (Beograd) (N.S.) 32(46) (1982), 149-153.

[12] M.R. Singh, Y. Mahendra Singh, Fixed points for biased maps on metric space, Int. J. Contemp. Math. Sci. Vol. 4 (2009), no. 16, 769-778. 
